\title{
Towards a Semantic Modelling Framework in Support of Multimodal User Interface Design
}

\author{
Elena Tsiporkova, Tom Tourwé, and Nicolás González-Deleito \\ Sirris - Software Engineering \& ICT Group, \\ A. Reyerslaan 80 - 1030 Brussels - Belgium \\ \{elena.tsiporkova, tom.tourwe, nicolas.gonzalez\} esirris.be
}

\begin{abstract}
In this paper, we propose a semantic modelling framework to capture the available domain knowledge in the field of multimodal interface design and to support designers in their daily design tasks.
\end{abstract}

\section{Introduction}

Parallel with the evolution towards multimodal applications, the research toward the establishment of formal principles and guidelines for multimodal interaction design is gaining increasing interest and importance in recent years (e.g. $[1,3,4])$. However, as observed by Sarter [5], the existing guidelines mostly focus on high-level design objectives and do not provide support on how to map them to the needs of an actual application. They do not capture and reflect on the considerable practical experience and valuable expert knowledge that interface designers rely on during their daily activities. Moreover, a considerable gap exists between the theory (formal guidelines) and the practice of multimodal human interface design, as different experts might approach the same interface design tasks in different ways based on personal expertise, background and intuition.

Our aim in this article is to work toward bridging this gap via the application of semantic technologies (e.g. ontologies) for capturing the available domain knowledge in the field of multimodal interface design. There are several advantages associated with such an approach: it guarantees a uniform approach across different designers within the same organisation, allows for semantic inter-usability of the formal guidelines across different applications and domains, and is open to allow for knowledge evolution and growth.

\section{Multimodal User Interface Design: Current Practices and Challenges}

A recent survey [1] on the current state-of-the-art of multimodal interfaces covers exhaustively the foundations and features of multimodal interaction, current developments in modelling languages and programming frameworks, and existing principles and guidelines for multimodal interface design. Several authors worked on establishing formal principles for multimodal user interface design. Sarter [5] reviewed the existing design guidelines for multimodal information presentation, 
approaching the problem from the point of view of main decisions and considerations involved in multimodal interface design. She identified four themes of guidelines: 1) the selection of modalities; 2) mapping modalities to tasks and types of information; 3 ) the combination, synchronisation and integration of modalities; 4) adaptation of multimodal information input and presentation to accommodate changing task contexts and circumstances.

Sarter argued that most shortcomings in the aforementioned guidelines are due to the fact that there remain a substantial number of open research problems in the area of multimodal interaction design. However, we consider that guidelines resulting from research would always remain of a rather conceptual and less empirical nature and thus of little practical use. In our opinion, an extensive intuitive and empirical knowledge base exists already within the designer community, and the challenge is to develop methods, formal languages and frameworks that allow for capturing and exploiting this knowledge. The semantic modelling framework proposed in this work is an initial attempt in this direction. The selected approach is based on Sarter's insight of considering the interface design process from the perspective of the decision dilemmas designers face daily when executing their design tasks.

\section{Semantic Modelling Framework}

Our semantic modelling framework allows us to capture general domain knowledge and expert knowledge, the latter is referring to Human-Computer Interaction (HCI) knowledge related to multimodal application design. Both domain and expert knowledge are described via an ontology, a formal representation of knowledge by a set of key domain concepts and the relationships between those concepts.

\subsection{Competency Questions and Domain Concepts}

To determine the scope of an ontology and identify the questions that an ontologybased knowledge repository should be able to answer, we need to state so-called competency questions [2]. Subsequently, from these questions the information that needs to be contained within the ontology, i.e. the concepts and the relationships between concepts, can be derived.

We consider that at this stage of the research, our ontology should be able to answer the following competency questions, which are inspired by the major themes identified by Sarter [5] (see above): 1) Which input and output modalities are available to the user of an application? 2) What are the different factors that affect the use of particular input and output modalities? 3) What are the appropriate (combinations of) modalities to support users in a particular task?

Based on this list of questions, the ontology will include information on users, their context and tasks, the devices they use and their specific capabilities, and usage characteristics and constraints of particular user-interface modalities.

Fig. 1 shows the high-level domain concepts of our ontology. For instance, the class User represents a user of an application, which itself is represented by the class Application. A class Location represents the physical location where the user is performing his activities and using the application. Locations are considered as being 
an IndoorLocation or an OutdoorLocation. The class Activity represents the activities that a user can engage in, subdivided into PrimaryActivity and SecondaryActivity.

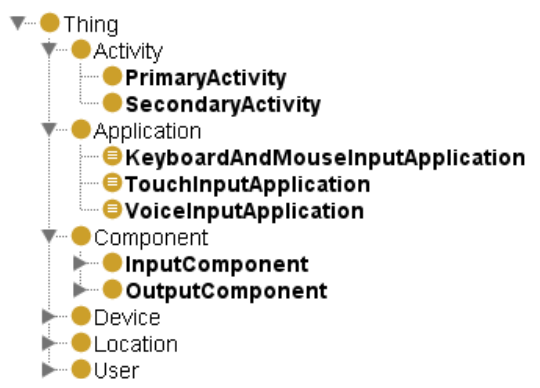

Fig. 1. High-level domain concepts in the semantic modelling framework

These concepts are related through relationships. Some relationships are defined through property chains which enable them to be automatically inferred from other relationships. For example, if we know that application $A$ is used by user $U$, and that this user is located in location $L$, we can infer that application $A$ is used in location $L$. More formally, A used_by $U \wedge U$ located_in $L \rightarrow A$ used_in $L$.

\subsection{Modelling Domain Knowledge and Design Guidelines}

We model domain knowledge by defining subclasses for the key domain concepts, specifying necessary and sufficient conditions as appropriate. We consider domain knowledge to be any factual information about users, applications and devices that potentially influences the decision about which modality to provide. This includes obvious information such as the specific input/output capabilities of a device, but also information such as physical and social aspects of the user's working environment, or particular aspects of the nature of the activity (e.g. primary and secondary tasks). For example, we consider that Microphone is a subclass of VoiceInputComponent which in itself is a subclass of InputComponent, and that PrivateWorkingSpace is an IndoorLocation that is quiet (by adding a condition has_noise_level some Quiet).

Design guidelines capture the expertise and experience of the HCI practitioners. They describe applicability conditions and constraints for the use of a particular multimodal interface. For instance, interaction through a vocal command can be suggested if the following conditions and constraints are met: 1) the application runs on a device that has access to a microphone; 2) the application is used in an environment with limited noise, since the accuracy of voice technology is heavily dependent on environmental noise conditions (e.g. background noise); 3) the application is used in a private environment, since controlling an application by voice might disturb surrounding colleagues; 4) the user only uses the application as a support for his primary activities which require the use of both hands, i.e. the application itself is not the primary focus of the user. 
We model this by introducing a subclass VoiceInputApplication of Application, representing applications that could use vocal commands, and by defining a necessary and sufficient condition representing the above conditions as follows:

(has_access_to some VoiceInputComponent) and (used_in some PrivateWorkingSpace) and (used_while_performing some (PrimaryActivity that requires some BothHands))

\section{Conclusions}

This paper presents an initial attempt to formally model and exploit relevant domain knowledge and expertise in support of selecting appropriate modalities during the human machine interface design process. The work presented here is inspired and results from our interactions with HCI practitioners. Besides further refinement of the presented semantic modelling framework, future research includes considering other competency questions that our ontology could support, expanding toward broad range of working contexts and types of users, and supporting synchronisation of modalities in time. All this research will be performed in close collaboration with HCI practitioners.

Acknowledgements. This research is supported by the ARTEMIS JU and IWT (www.iwt.be) through the SMARCOS project (www.smarcos-project.eu), and by Innoviris (www.innoviris.be) through the VariBru project (www.varibru.be).

\section{References}

1. Dumas, B., Lalanne, D., Oviatt, S.: Multimodal interfaces: A survey of principles, models and frameworks. Human Machine Interaction, 3-26 (2009)

2. Gruninger, M., Fox, M., et al.: Methodology for the Design and Evaluation of Ontologies. In: Proceedings of the Workshop on Basic Ontological Issues in Knowledge Sharing, IJCAI, vol. 95 (1995)

3. Oviatt, S.: Multimodal interfaces. In: The Human-computer Interaction Handbook: Fundamentals, Evolving Technologies and Emerging Applications, pp. 286-304 (2003)

4. Reeves, L., Lai, J., Larson, J., Oviatt, S., Balaji, T., Buisine, S., Collings, P., Cohen, P., Kraal, B., Martin, J., et al.: Guidelines for multimodal user interface design. Communications of the ACM 47(1), 57-59 (2004)

5. Sarter, N.: Multimodal information presentation: Design guidance and research challenges. International Journal of Industrial Ergonomics 36(5), 439-445 (2006) 